\title{
A case report: Isolated Liver Tuberculosis
}

\author{
Ruksana Karim ${ }^{1}$, Md Mohsen Chowdhury ${ }^{2}$, Arif Salam Khan ${ }^{3}$ \\ ${ }^{1}$ Professor Department of Biochemistry, Uttara Adunik Medical college and Hospital ${ }^{2}$ Professor, Hepatobiliary surgery, Deprtment of surgery, BSMMU ${ }^{3}$ Assistant Professor, Department of Surgery, \\ BSMMU.
}

\begin{abstract}
:
Isolated liver tuberculosis is still considered a rare condition and its atypical clinical presentation challenges the clinical acumen of the treating physician. There is difficulty in reaching the correct preoperative diagnosis of nodular hepatic tuberculosis that presents as a space-occupying lesion. It is usually unsuspected and confused with primary or metastatic carcinoma of the liver. In this report, we describe a rare case of isolated liver tuberculosis.
\end{abstract}

Key words: Liver granuloma, Liver tuberculosis

[BSMMUJ 2014; 7 (1) : 71-73]

\section{Introduction:}

Tuberculosis remains an important public health problem in Bangladesh. Isolated liver tuberculosis (ILT) is still considered a rare condition and hepatic tuberculosis is usually associated with an active pulmonary or miliary tuberculosis. ${ }^{1,2}$ Liver involvements in tuberculosis is usually clinically silent. Isolated hepatic tuberculosis is the rarest form of local hepatic tuberculosis. ${ }^{3}$ Tuberculosis presenting as an isolated liver tumor, without active pulmonary or miliary tuberculosis, or other clinical evidence of tuberculosis is distinctly rare. ${ }^{4}$ In this report, we describe a rare case of isolated liver tuberculosis.

\section{Case Report:}

A 48-year-old femals was admitted in Hepatobiliary surgery division BSMMU with right upper-abdominal pain and weight loss for six months and occasional fever. The patient's vital signs were stable. Physical examination showed epigastric tenderness without hepatomegaly. Laboratory data revealed normal serum hemoglobin level, normal white blood cell count with, normal erythrocyte sedimentation rate, normal liver and renal function tests, and normal coagulation tests. Tumor markers alpha-

Address for Correspondence: Md. Mohsen Chowdhury, FCPS Professor Hepatobiliary surgery, Department of surgery, BSMMU

Mobile no:-01819 228708, Email: drmohsen12@hotmail.com fetoprotein, CEA, CA 19-9 and CA-125 were normal. There was no radiological finding of tuberculosis in the X-ray chest. Liver ultrasonography showed multiple hypodense lessions in both lobes of the liver. Computed tomography of the abdomen showed a multicystic lesion involving both lobes of the liver (Figures 1-2). Upper GIT and colonoscopy were normal.

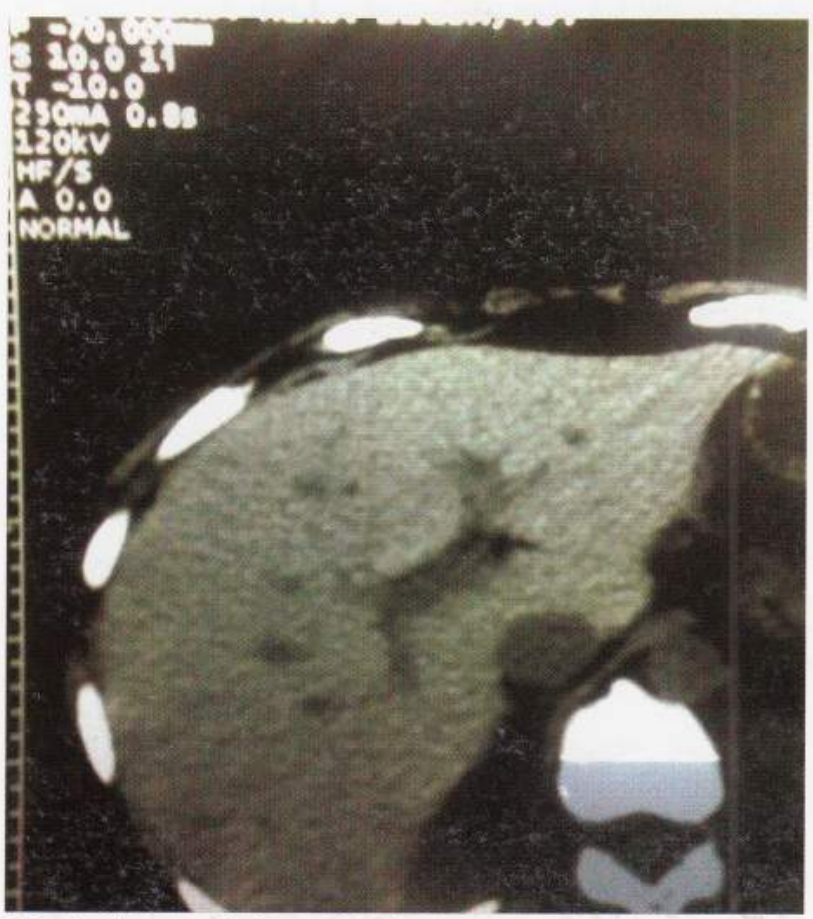

Fig-1: An axial CT scan showing a multiple hypodense areas in both lobes of the liver. 


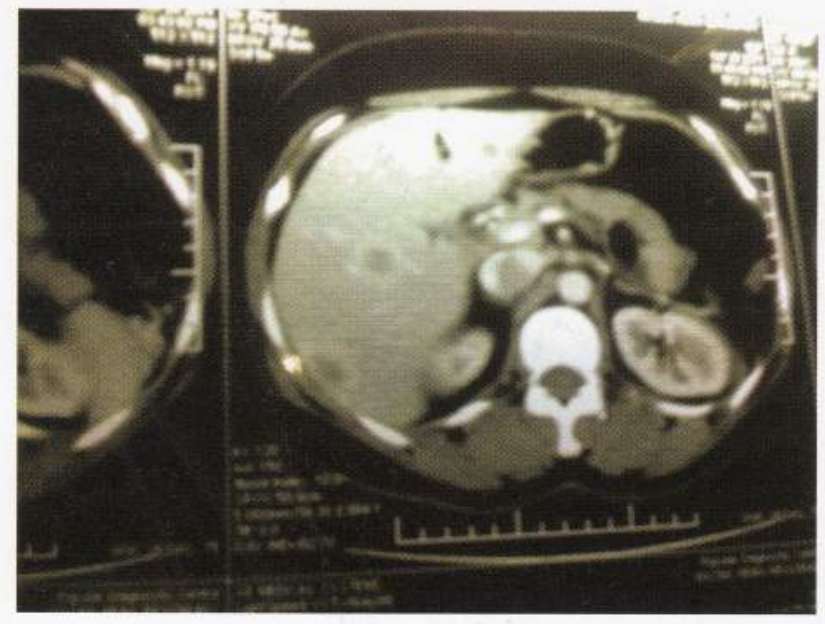

Fig-2: A CT scan showing a hypo dense mass without clear margins within the right lobe of the liver.

A Percutaneous tru-cut biopsy of the liver revealed areas of caseous necrosis and classic tubercles.

The patient was discharged. Isoniazid $300 \mathrm{mg} /$ day, rifampicin $600 \mathrm{mg} /$ day, pyrazinamide $1500 \mathrm{mg} /$ day and ethambutol $1500 \mathrm{mg} /$ day were administered for two months and isoniazid $300 \mathrm{mg} /$ day and rifampicin $600 \mathrm{mg} /$ day were subsequently administered for four months. After treatment, the patient was followed up for eight months without encountering any problem. Follow up USG and CT scan were done 2 it was normal done.

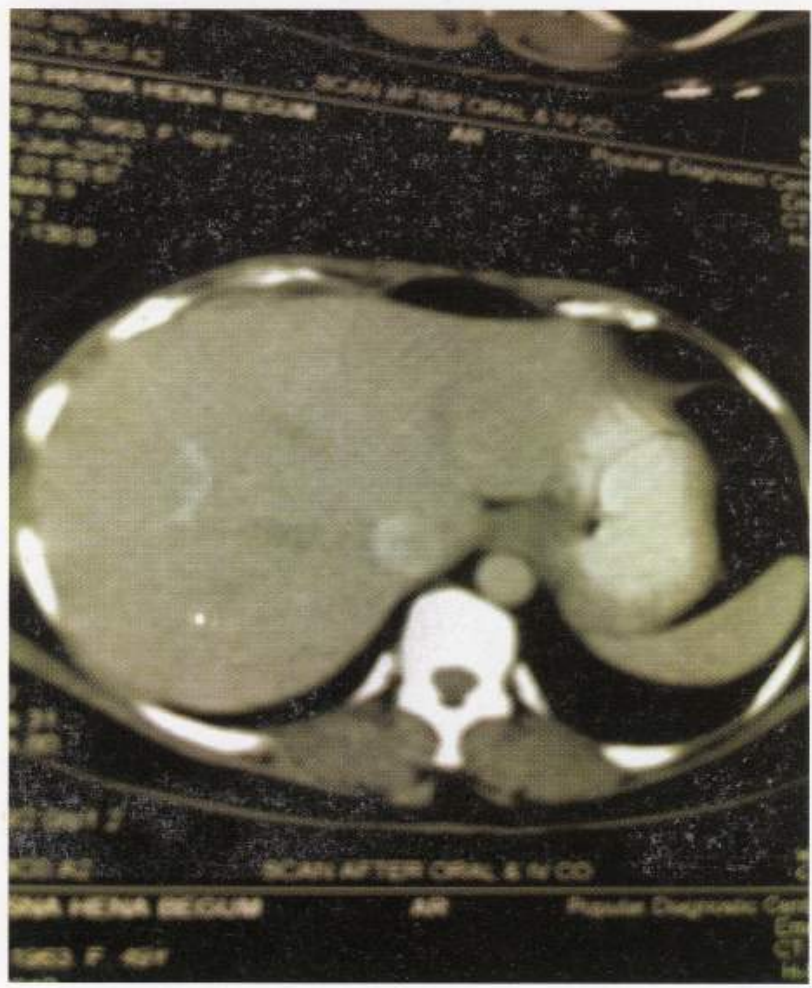

Fig-3: CT scan done after six months.

\section{Discussion:}

There are three forms of hepatic tuberculosis. Diffuse hepatic involvement with pulmonary or miliary tuberculosis is the most common form seen in $50 \%$ to $80 \%$ of patients dying of pulmonary tuberculosis. Diffuse hepatic infiltration without recognizable pulmonary involvement is the second form. The third very rare form presents as a focal/local tuberculoma or abscess. ILT the rarest form of local hepatic tuberculosis. ${ }^{5}$ Kok et $\mathrm{al}^{6}$ reported an overall incidence of $0.3 \%$ for isolated hepatic tuberculosis. Hepatic tuberculosis lesions that appear as masses larger than $2 \mathrm{~mm}$ in diameter are referred to as macronodular and pseudotumoural tuberculosis. On the basis of imaging examinations alone, these lesions are virtually indistinguishable from many other focal lesions of the liver, such as hepatocellular carcinoma, metastases and Hodgkin's disease, so pathological examination is necessary for diagnosis. 3

Isolated hepatic tuberculosis results from tubercle bacilli gaining access to the portal vein from a microscopic or small tubercular focus in the bowel. The clinical presentation of ILT is so rare and atypical that it challenges the clinical acumen of the treating physician. 2 The difficulty is reaching a correct preoperative diagnosis of nodular hepatic tuberculosis that presents as a space-occupying lesion. It is usually unsuspected and confused with primary or metastatic carcinoma of the liver, as in our case. Radiological findings of hepatic tuberculosis are not specific although multiple hypodense lesions have been described on $\mathrm{CT}$ scan in cases of macronodular tuberculoma of the liver.7 The radiologic diagnosis of hepatic tuberculoma is difficult and histopathologic diagnosis is required to distinguish tuberculosis from lymphoproliferative disorder, metastatic deposits and other granulomatous disease like sarcoidosis and fungal infection. Establishing the diagnosis is not easy, especially if there is no history of previous tubeculosis exposure. The definitive diagnosis could be done with tests on histological and bacteriological tuberculosis in liver biopsy specimens. The importance of establishing the correct diagnosis cannot be overstated, since untreated evidence of tubercu- 
sary for diagnosis. ${ }^{3}$

Isolated hepatic tuberculosis results from tubercle bacilli gaining access to the portal vein from a microscopic or small tubercular focus in the bowel. The clinical presentation of ILT is so rare and atypical that it challenges the clinical acumen of the treating physician. ${ }^{2}$ The difficulty is reaching a correct preoperative diagnosis of nodular hepatic tuberculosis that presents as a space-occupying lesion. It is usually unsuspected and confused with primary or metastatic carcinoma of the liver, as in our case. Radiological findings of hepatic tuberculosis are not specific although multiple hypodense lesions have been described on CT scan in cases of macronodular tuberculoma of the liver. ${ }^{7}$ The radiologic diagnosis of hepatic tuberculoma is difficult and histopathologic diagnosis is required to distinguish tuberculosis from lymphoproliferative disorder, metastatic deposits and other granulomatous disease like sarcoidosis and fungal infection. Establishing the diagnosis is not easy, especially if there is no history of previous tubeculosis exposure. The definitive diagnosis could be done with tests on histological and bacteriological tuberculosis in liver biopsy specimens. The importance of establishing the correct diagnosis cannot be overstated, since untreated evidence of tuberculosis. The histological picture of hepatic tuberculoma is usually that of a large epitehloid tumour composed of conglomerate tubercles with central caseation necrosis. Langerhans-type gaint cells may be found in the granuloma and are surrounded by lymphohistiocytic cells, plasma cells and eosinophils. ${ }^{8}$ In view of the nonspecific presentation and imaging appearance of the disease, a high index of suspicion is required to obtain a preoperative diagnosis. ${ }^{9}$ In this case, the diagnosis was established by CT guided biopsy. . Ultrasound-guided percutaneous liver biopsy, CT and laparoscopy are adequate methods of obtaining tissue for diagnosis. The presence of caseating granuloma is usually sufficient to establish the diagnosis. If the diagnosis is still in doubt, laparoscopy is the next investigative method of choice, as it is less invasive than laparotomy. Clinicians' reliance on laparotomy and the procedure's utilization rate is an indication of the difficulty of diagnosis.$^{10,11}$
In conclusion, preoperative diagnosis of isolated liver tuberculosis that presents as space occupying lesions is difficult. It is mostly confused with primary or metastatic carcinoma of the liver

\section{References:}

1. Zulfiqur RK, Mohsen MC, M S Uddin, M A Taher .A Case Report: Liver Tuberculosis: BSMMU J 2009; 2(2): 88-9.

2. Yağmu RY, Girgin S, E. Gedik, H. Büyükbayram: A case report: Isolated Liver Tuberculosis. The Internet Journal of Surgery. 2009 ; 19 : 1. DOI: $10.5580 / 418$

3. Bangroo AK, Malhotra AS. Isolated hepatic tuberculosis. JAPS 2005; 10: 105-7.

4. Singh D, Singh S, Raut SB, Karmarkar SJ. Isolated liver tuberculosis: a case report. Pediatr Surg Int 2004; 20: 727-28.

5. Vimalraj V, Jyotibasu D, Rajendran S et al.Macronodular hepatic tuberculosis necessitating hepatic resection: a diagnostic conundrum. Can J Surg 2007; 10;50: E7-8

6. Nampoory MR, Halim MM, Shreedharan R, al-Sweih NA, Gupta RK, Costandi JN. Liver abscess and disseminated intravascular coagulation in tuberculosis. Postgrad Med J 1995;71:490-92.

7. Purl AS, Nayyar AK, Vij JC. Hepatic tuberculosis. Ind J Tub 1994; 41: 131-34.

8. Kok KY, Yapp SK. Isolated hepatic tuberculosis: report of five cases and review of the literature. J Hepatobiliary Pancreat Surg 1999; 6: 195-98.

9. Kumar V, Pandey D. Isolated hepatosplenic tuberculosis. Hepatobiliary Pancreat Dis Int 2008; 7: 328-30

10. Tan TC, Cheung AY, Wan WY, Chen TC. Tuberculoma of the liver presenting as a hyperechoic mass on ultrasound. Br J Radiol 1997; 70: $1293-5$.

11. Debnath PR, Tripathi R, Kandpall D, Kumar B, Malik E, Sharma $\mathrm{SB}$. Isolated tubercular liver abscess in children treated with percutaneous isoniazid infusion. Indian J Tuberc 2007; 54: $149-51$.

12. Chen HC, Chao YC, Shyu RY, Hsieh TY. Isolated tuberculous liver abscesses with multiple hyperechoic masses on ultrasound: a case report and review of the literature. Liver Int 2003; 23: $346-50$

13. Alcantara-Payawal DE, Matsumura M, Shiratori $\mathrm{Y}$, Okudaira $\mathrm{T}$, Gonzalez R, Lopez RA, Sollano JD, Omata M. Direct detection of Mycobacterium tuberculosis using polymerase chain reaction assay among patients with hepatic granuloma. J Hepatol 1997; 27: $620-27$. 